\title{
Acamprosate and baclofen were not effective in the treatment of pathological gambling: preliminary blind rater comparison study
}

\section{Pinhas N. Dannon*, Oded Rosenberg, Netta Schoenfeld and Moshe Kotler}

Beer Yaakov Mental Health Center affiliated to Tel Aviv University, Beer Yaakov, Israel

\section{Edited by:}

Michel Lejoyeux, Assistance Publique Hôpitaux de Paris Université Paris

Diderot, France

\section{Reviewed by:}

Laurent Karila, Université Paris Sud,

France

Candice Germain, Hopital Beaujon,

France

*Correspondence:

Pinhas N. Dannon, Research

Department, The Beer Yaakov Mental

Health Hospital, Beer Yaakov,

P.O. Box 1, Israel.

e-mail: pinhasd@post.tau.ac.il

\begin{abstract}
Objectives: Pathological gambling (PG) is a highly prevalent and disabling impulse control disorder. A range of psychopharmacological options are available for the treatment of PG, including selective serotonin reuptake inhibitors, opioid receptor antagonists, anti-addiction drugs, and mood stabilizers. In our preliminary study, we examined the efficacy of two anti-addiction drugs, baclofen and acamprosate, in the treatment of PG. Materials and Methods: Seventeen male gamblers were randomly divided into two groups. Each group received one of the two drugs without being blind to treatment. All patients underwent a comprehensive psychiatric diagnostic evaluation and completed a series of semi-structured interviews. During the 6-months of study, monthly evaluations were carried out to assess improvement and relapses. Relapse was defined as recurrent gambling behavior. Results: None of the 17 patients reached the 6-months abstinence. One patient receiving baclofen sustained abstinence for 4 months. Fourteen patients succeeded in sustaining abstinence for 1-3 months. Two patients stopped attending monthly evaluations. Conclusion: Baclofen and acamprosate did not prove efficient in treating pathological gamblers.
\end{abstract}

Keywords: pathological gambling, behavioral addiction, baclofen, acamprosate, GABA

\section{INTRODUCTION}

Pathological gambling (PG) is classified in the Diagnostic and Statistical Manual of Mental Disorders, Fourth Edition, as an impulse control disorder (American Psychiatric Association, 2000). In the International Classification of Diseases of the World Health Organization, $\mathrm{PG}$ is considered a habit and impulse disorder, alongside kleptomania; trichotillomania, and pyromania, all of which are characterized by an overwhelming urge to perform a harmful act (World Health Organization, 1992). PG is a chronic, progressive, male-dominant disorder, causing great personal and social consequences such as suicide attempts, job loss, martial and family problems, legal problems, and criminal behaviors (Dannon et al., 2006; Iancu et al., 2008).

To date, a range of psychopharmacological options are available for the treatment of PG, including selective serotonin reuptake inhibitors (SSRI), opioid receptor antagonists, anti-addiction drugs, and mood stabilizers.

Multiple randomized, double-blind as well as open-label shortterm $(8,12$, or 16-week) trials have assessed pharmacotherapy for the acute treatment of PG. As a group, these trials show an overall mixed success with several double-blind studies failing to show short-term efficacy. To date, there have been four doubleblind trials of SSRIs for the treatment of PG. While two studies showed short-term efficacy (Hollander et al., 2000; Kim et al., 2002), separate studies by Blanco et al. (2002) and Grant et al. (2003) found no significant statistical superiority of the SSRI agent when compared to a placebo. Preliminary studies have examined the use of the mood stabilizers, topiramate (Dannon et al., 2005a), and anti-addiction drug, bupropion (Black, 2004; Dannon et al., 2005b,c), for the short-term treatment of PG with most studies showing a beneficial effect. In addition, the anti-addiction, opioid antagonist naltrexone, has been shown to be beneficial in treating the cravings and urges associated with PG (Kim and Grant, 2001; Kim et al., 2001).

It is well accepted in the clinical literature of PG that there are several important limitations of existing short-term medication trials: (1) variable rates of placebo response have been demonstrated in the short-term studies, raising the question of whether improvement seen in treatment groups may be related to nonmedication mechanisms of action; (2) PG typically has a chronic course, and therefore acute remission of symptoms may not lead to a clinically significant improvement in functioning if the remission is not sustained; and (3) in all short-term studies published to date, remission of gambling symptoms was judged, at least in part, according to patients' self-reports of gambling behavior which may be biased (Dannon et al., 2007).

The opioid system controls the processing of reward, pleasure, and pain, alongside gambling-related urges. Data suggests that opioid receptor antagonists mediate their therapeutic action in the treatment of addictive disorders through the modulation of GABA neuronal input to dopamine neurons in the mesolimbic pathway (Kim and Grant, 2001; Kim et al., 2001). GABA represents the main inhibitory neurotransmitter in the brain. High levels of $\mathrm{GABA}_{\mathrm{B}}$ receptor expression in the limbic system indicate a role in regulating emotional behavior (Addolorato et al., 2009). The assumption is being that the GABA receptors may play a role 
in the pattern of urges, craving, and sense of enjoyment seen in PG behavior (Addolorato et al., 2009).

The brain's reward circuit, located in the nucleus accumbens, ensures reinforcement of behaviors associated with species survival and procreation. The opioid system is involved in the process of reward, pleasure, pain, and gambling-related urges. It has been suggested that opioid receptor antagonists mediate their therapeutic action in the treatment of addictive disorders through the modulation of GABA neuronal input to dopamine neurons in the mesolimbic pathway (Addolorato et al., 2009). The above data had led us to believe that GABA directed addiction drugs, such as baclofen and acamprosate, might be efficient in the treatment of PG.

Alcohol has been proven to affect GABA neurotransmitters in the brain, and anti-addiction drugs have been shown to be efficient in decreasing the consumption and craving of alcohol. Acamprosate is believed to increase the effect on the inhibitory neurotransmitter GABA and to decrease excitatory glutamate activity at NMDA receptors (Addolorato et al., 2009). Baclofen is known to suppress alcohol-stimulated dopamine release in the shell of the nucleus accumbens of rats (Addolorato et al., 2009), and its administration has been shown to promote alcohol abstinence and reduce alcohol craving and intake; it has also been shown to reduce anxiety (Addolorato et al., 2006).

Previous experience with anti-addiction medications, such as bupropion and naltrexone, has been shown to be the most effective for the treatment of PG (Kim and Grant, 2001; Kim et al., 2001; Black, 2004; Dannon et al., 2005b,c). Based on the effectiveness of anti-addiction drugs, we examined the efficacy of two other medications, baclofen and acamprosate. The difference between the two groups is the system on which the drug affects; while bupropion and naltrexone affect the reward system and the neurotransmitter dopamine, the baclofen, and acamprosate could affect the GABA system.

In our study, we tested the efficacy of two addiction drugs, acamprosate and baclofen, which stimulate the GABA receptors, and have been proven to be effective in the treatment of alcohol abuse (Addolorato et al., 2009).

\section{Acamprosate}

Acamprosate (calcium acetylhomotaurinate) is a simple derivative of the essential taurine amino acid which displays a structural resemblance to gamma-amino butyric acid (GABA; American Psychiatric Association, 2000). It was approved by the US FDA in 2004 for treatment of alcoholic patients to decrease alcohol craving after alcohol detoxification. Acamprosate seems to bind specifically to $\mathrm{GABA}_{\mathrm{B}}$ receptors (Boothby and Doering, 2005). It enhances GABA reception and the transmission of the GABAergic system, raises the continuous alcohol abstinence rate and doubles the days of cumulative abstinence from alcohol (Boothby and Doering, 2005). Acamprosate is not a sedative; it does not possess addictive properties or provide reinforcing effects.

2 Baclofen

Baclofen [beta-(4-chlorophenyl)-GABA] is a $\mathrm{GABA}_{\mathrm{B}}$ receptor agonist that has been found to suppress both acquisition of alcohol drinking behaviors in rats and daily alcohol intake in alcohol experienced rats. It also suppresses alcohol-stimulated dopamine released in the shell of the nucleus accumbens of rats (Addolorato et al., 2009).

In alcohol dependant subjects, baclofen administration promotes alcohol abstinence, induces the remission of withdrawal symptoms, and reduces alcohol craving and alcohol intake (Addolorato et al., 2006).

\section{MATERIALS AND METHODS STUDY SUBJECTS}

Patients were referred to our clinic, from ambulatory services throughout Israel, on account of our gambling disorder expertise. All study patients signed an informed consent form after possible side effects of the study medications were explained to them. Current study was approved by local IRB committee to publish the results of clinical trial. Patients also gave consent for family members to be interviewed at the monthly follow-up visits. Inclusion criteria included: PG diagnosis according to DSM-IV-TR criteria and South Oaks gambling scale (SOGS) score of more than 5. Exclusion criteria included: co-morbidity with axis one psychiatric disorders, neurological disorders, alcohol and substance abuse, and treatment with any psychiatric medication in the month before to the screening interview.

All patients underwent a comprehensive psychiatric diagnostic evaluation and completed a series of semi-structured interviews performed by a senior psychiatrist (PND).

Study subjects were evaluated on a monthly basis throughout the duration of the study in order to assess measures of sustained improvement (i.e., abstinence) and relapse. In this study, abstinence was strictly defined as no gambling behavior (including any form of gambling) during the month preceding the follow-up visit. Relapse was defined as any gambling behavior during the month preceding the follow-up visit.

\section{Instruments}

We administered the Hamilton rating scale for anxiety (HARS; Hamilton, 1959), the Hamilton depression rating scale (HDRS; Hamilton, 1959, 1960), visual analog scale (VAS; Guy, 1976), and the South Oaks gambling screen (SOGS; Leisure and Blume, 1987) at baseline. HARS, HDRS, VAS were administered at every monthly follow-up visit. The patients' self-reports regarding amount of time spent gambling was assessed at each follow-up visit although a structured interview was not performed. Collateral information regarding gambling behavior was collected from family members at the monthly follow-up visits. All scales were administered by the rater (TL) who was blind to drug treatment.

\section{MEDICATION}

Patients were randomly assigned to treatment with baclofen or acamprosate. Treatment with baclofen was conducted as follows: $10 \mathrm{mg}$ per day for the first 4 days, than $20 \mathrm{mg}$ for the next 4 days, and than $30 \mathrm{mg}$ with stable condition up to 3 weeks. Average dosage of baclofen was $30 \mathrm{mg}$ per day $(\mathrm{SD}+7.5)$. Some patients were treated with up to $50 \mathrm{mg}$ per day.

Treatment with acamprosate was conducted as follows: $333 \mathrm{mg}$ for the first 4 days than $666 \mathrm{mg}$ for a week and afterward $999 \mathrm{mg}$ per day. Average dosage of acamprosate was $666 \mathrm{mg}(\mathrm{SD}+174)$. 


\section{Analysis}

Statistical analysis was performed with $t$-test and ANOVA analyses. Levels of significance were set at 0.05 , unless otherwise stated. Also, two tailed $t$-test was chosen to perform a part of the statistical analysis for independent samples.

\section{RESULTS}

Patients' ages ranged between 19 and 69 years $(29.6 \pm 16.5)$. Demographic data are summarized in Table 1.

The average SOGS score was $7.4+2.8$ at baseline visit in two groups. Also, HDRS, HARS, and VAS average scores were similar and summarized in Table 2.

According to treatment regimens $T$-test comparison has shown no statistical differences in HDRS, HARS, or VAS (Table 3).

Also, ANOVA analyses of VAS scores of patients treated with either medication at baseline, after 1 month and at relapse has

Table 1 | Demographic characteristics of the patients.

\begin{tabular}{|c|c|c|c|}
\hline & $\begin{array}{l}\text { Baclofen } \\
(N=9)\end{array}$ & $\begin{array}{l}\text { Acamprosate } \\
(N=8)\end{array}$ & $\begin{array}{l}P \\
\text { values }\end{array}$ \\
\hline Age & $29.7 \pm 13.5$ & $30.4 \pm 11.9$ & NS \\
\hline \multicolumn{4}{|l|}{ Ethnicity \% } \\
\hline North African decent & 46 & $50 \%$ & NS \\
\hline Eastern European decent & $30 \%$ & $24 \%$ & NS \\
\hline Israeli born & $24 \%$ & $26 \%$ & NS \\
\hline \multicolumn{4}{|l|}{ Education \% } \\
\hline 12th grade & $40 \%$ & $40 \%$ & NS \\
\hline High School diploma & $36 \%$ & $40 \%$ & NS \\
\hline University & $24 \%$ & $20 \%$ & NS \\
\hline \multicolumn{4}{|l|}{ Employment \% } \\
\hline Unemployed & $18 \%$ & $16 \%$ & NS \\
\hline Full/part time & $82 \%$ & $84 \%$ & NS \\
\hline \multicolumn{4}{|l|}{ Marital status \% } \\
\hline Married & $70 \%$ & $66 \%$ & NS \\
\hline Widowed-divorced-separated & $18 \%$ & $20 \%$ & NS \\
\hline Never married & $12 \%$ & $14 \%$ & NS \\
\hline
\end{tabular}

Table 2 | Statistical comparison of HDRS, HARS, and VAS scores of patients treated with acamprosate and baclofen.

\begin{tabular}{llll}
\hline & $\begin{array}{l}\text { Visual } \\
\text { analog } \\
\text { scale }\end{array}$ & $\begin{array}{l}\text { Hamilton } \\
\text { depression } \\
\text { rating } \\
\text { scale }\end{array}$ & $\begin{array}{l}\text { Hamilton } \\
\text { anxiety } \\
\text { rating } \\
\text { scale }\end{array}$ \\
\hline $\begin{array}{l}\text { Unpaired T-TEST } \\
\text { at baseline }\end{array}$ & $P>0.74$ & $P>0.86$ & $P>0.78$ \\
$\begin{array}{l}\text { Unpaired T-TEST } \\
\text { after 1 month of } \\
\text { treatment } \\
\text { completion } \\
\text { Unpaired } T \text {-TEST } \\
\text { at the time of } \\
\text { relapse }\end{array}$ & $P>0.73$ & $P>0.9$ & $P>0.1$ \\
\hline
\end{tabular}

HDRS, Hamilton depression rating scale; HARS, Hamilton anxiety rating scale; VAS, visual analog scale. shown no statistical differences $(P=0.9 ; f=0.3$; lambda $=1.5$; power $=0.1$ ).

ANOVA analyses of HDRS scores of patients treated with either medication at baseline, after 1 month and at relapse has shown no statistical differences $(P=0.92 ; f=0.26$; lambda $=1.3$; power $=0.1$ ).

ANOVA analyses of HARS scores of patients treated with either medication at baseline, after 1 month and at relapse has shown no statistical differences $(P=0.38 ; f=1.1$; lambda $=5.4$; power $=0.3$ ).

\section{SIDE EFFECTS}

Baclofen induced side effects were: weakness, apathy, headaches, nausea. Acamprosate induced side effects included: nausea, vomiting head aches, muscle aches, stiffness. None of the patients stopped the medication due these mild side effects.

\section{DISCUSSION}

None of the subjects from both groups succeeded to complete the 6-months of abstinence from gambling, we conclude that these two medications were not effective to stop the gambling behavior. However, recently published case report showed the effectiveness of acamprosate. In this case patient was addicted to alcohol and gambling (Raj, 2010).

We postulate that the GABA system, in oppose to the reward system, most likely, does not have a major role in manifesting gambling in the PG. Dannon et al. (2006) and Iancu et al. (2008) have reviewed PG literature and have suggested a comprehensive theory of PG typology and treatment ramifications of each PG subtype. Therefore, this model was further used in this study as it may well benefit treatment efficacy for PG.

Dannon et al. (Dannon et al., 2006; Iancu et al., 2008) identified a typology consisting of three PG subtypes: (1) addictive PG, which is the largest subtype characterized by moderate severity of PG, increase alcohol abuse and dependence and being predominantly male. This subtype is suggested to best benefit from opioid antagonists and anti-addiction drugs, (2) an obsessive compulsive PG which is characterized by being predominantly female gamblers; onset tends to be in midlife in response to a perceived psychological trauma, increased mood and anxiety co-morbidity. These patients may best benefit from anti-depressants such as SSRIs and SNRIs and from psychotherapy addressing stress resolution and coping mechanisms, and (3) an impulsive PG subtype characterized by high levels of risk-taking behaviors, little ability to plan and a tendency to spend large sums of money at one sitting. This subtype tends to present with alcohol abuse, attention deficit disorder (ADD) and other impulse control disorders. These patients may respond best to lithium or other mood stabilizers/antiepileptic drugs thought to target impulsive behavior.

The hypothetical model of sub typing is to explain the effectiveness of different medications through different subtypes of PG. In this preliminary study may have involved addicted subtype of pathological gamblers but, may be the small sample size, could not present equally all subtypes. However, the relatively low levels of the patients ability to stop the gambling behavior more than a month period demonstrates the ineffectiveness of these to drugs in the treatment of gambling addiction. 
Table 3 | Average scores and SD of HDRS, HARS, and VAS.

Baclofen

\begin{tabular}{|c|c|c|c|c|c|c|}
\hline & & \\
\hline & Baseline & After 1 month & At the time of relapse & Baseline & After 1 month & At the time of relapse \\
\hline HDRS & $10.1 \pm 2$ & $10.6 \pm 1$ & $10.6 \pm 1.5$ & $10.3 \pm 1.5$ & $10.7 \pm 1.7$ & $10.7 \pm 1.2$ \\
\hline HARS & $11.25 \pm 1.66905$ & $11.75 \pm 0.707$ & $11.9 \pm 0.64$ & $11 \pm 1.732$ & $10.9 \pm 1.21$ & $11.9 \pm 1.07$ \\
\hline VAS & $52.5 \pm 11.6$ & $51.8 \pm 10.3$ & $49.3 \pm 6.2$ & $54.3 \pm 8.4$ & $53.6 \pm 8.5$ & $52.8 \pm 6.3$ \\
\hline
\end{tabular}

HDRS, Hamilton depression rating scale; HARS, Hamilton anxiety rating scale; VAS, visual analog scale.

The importance of pharmacological preliminary studies such as our study ensues from the fact that no established pharmacotherapy exists. Potentially effective directions for future pharmacotherapy need to be explored on the basis of preliminary studies, or alternatively, as in this case, be renounced.

\section{LIMITATIONS}

The small sample size of this study limits the power of the study. Another limitation is patients were not blind to pharmacotherapy.
A third limitation was the lack of a medication-free placebo control group. However, since the results of this study indicate the inefficacy of both of the medications, this limitation does not lessen the strength of this particular study. We do recommend that further studies using a placebo-controlled design should be carried out in order to confirm our preliminary findings.

\section{ACKNOWLEDGMENTS}

Authors thank to Ms Tammy Lev for her participation in this study.

\section{REFERENCES}

Addolorato, G., Leggio, L., Agabio, R., Colombo, G., and Gasbarrini, G. (2006). Baclofen: a new drug for the treatment of alcohol dependence. Int. J. Clin. Pract. 60, 1003-1008.

Addolorato, G., Leggio, L., Cardone, S., Ferrulli, A., and Gasbarrini, G. (2009). Role of the GABA (B) receptor system in alcoholism and stress: focus on clinical studies and treatment perspectives. Alcohol 43, 559-563.

American Psychiatric Association. (2000). Diagnostic and Statistical Manual of Mental Disorders, 4th Edn. Washington, DC: American Psychiatric Association.

Black, D. (2004). An open-label trial of bupropion in the treatment of pathological gambling. J. Clin. Psychopharmacol. 24, 108-109.

Blanco, C., Petkova, E., Ibanez, A., and Sáiz-Ruiz, J. (2002). A pilot placebo-controlled study of fluvoxamine for pathological gambling. Ann. Clin. Psychiatry 14, 9-15.

Boothby, L. A., and Doering, P. L. (2005). Acamprosate for the treatment of alcohol dependence. Clin. Ther. 27, 695-714.

Dannon, P. N., Lowengrub, K., Gonopolski, Y., Musin, E., and Kotler, M. (2006). Pathological gambling: a review of phenomenological models and treatment modalities for an under recognized psychiatric disorder. Prim Care Companion J. Clin. Psychiatry 8, 334-339.
Dannon, P. N., Lowengrub, K., Musin, E., Gonopolski, Y., and Kotler, M. (2005a). Sustained release Bupropion versus naltrexone in the treatment of pathological gambling. J. Clin. Psychopharmacol. 25, 593-596.

Dannon, P. N., Lowengrub, K., Gonopolski, Y., Musin, E., and Kotler, M. (2005b). Topiramate versus fluvoxamine in the treatment of pathological gambling: a randomized, blind-rater comparison study. Clin. Neuropharmacol. 28, 6-10.

Dannon, P. N., Lowengrub, K., Musin, E., Gonoposki, Y., and Kotler, M. $(2005 c)$. Sustained release bupropion in the treatment of SSRI no responder pathological gamblers: pilot study and review of the literature. Therapy 2, 753-759.

Dannon, P. N., Lowengrub, K., Musin, E., Gonopolski, Y., and Kotler, M. (2007). 12-month follow-up study of drug treatment in pathological gamblers: a primary outcome study. J. Clin. Psychopharmacol. 27, 620624.

Grant, J. E., Kim, S. W., Potenza, M. N., Blanco, C., Ibanez, A., Stevens, L., Hektner, J. M., and Zaninelli. R. (2003). Paroxetine treatment of pathological gambling: a multicentre randomized controlled trial. Int. Clin. Psychopharmacol. 18, 243249.

Guy, W. (1976). ECDEU Assessment Manual for Psychopharmacology. Rockville, MD: US Department of Health, Education, and Welfare Publication (ADM), 76-338.
Hamilton, M. (1959). The assessment of anxiety states by rating. $\mathrm{Br}$. J. Med. Psychol. 32, 50-55.

Hamilton, M. (1960). A rating scale for depression. J. Neurol. Neurosurg. Psychiatr. 23, 56-62.

Hollander, E., DeCaria, C. M., Finkell, J. N., Begazab, T., Wongab, C. M., Cartwrightab, C. (2000). A randomized double-blind fluvoxamine/placebo crossover trial in pathologic gambling. Biol. Psychiatry 47, 813-817.

Iancu, I., Lowengrub, K., Dembinsky, Y., Kotler, M., and Dannon, P. N. (2008). Pathological gambling: an update on neuropath physiology and pharmacotherapy. CNS Drugs 22, 123-138.

Kim, S. W., Grant, J. E., Adson, D. E., Shin, Y. C., and Zaninelli, R. (2002). A double-blind placebo-controlled study of the efficacy and safety of paroxetine in the treatment of pathological gambling. J. Clin. Psychiatry 63, 501-507.

Kim, S. W., and Grant, J. E. (2001). An open naltrexone treatment study in pathological gambling disorder. Int. Clin. Psychopharmacol. 16, 285-289.

Kim, S. W., Grant, J. E., Adson, D. E., and Shin, Y. C. (2001). Double-blind naltrexone and placebo comparison study in the treatment of pathological gambling. Biol. Psychiatry 49, 914-921.

Leisure, H., and Blume, S. B. (1987). The South Oaks gambling screen (the SOGS): a new instrument for the identification of pathological gamblers. Am. J. Psychiatry 144, 1184 1188 .
Raj, Y. P. (2010). Gambling on acamprosate: a case report. J. Clin. Psychiatry 71, 1245-1246.

World Health Organization. (1992). The ICD-10 Classification of Mental and Behavioral Disorders: Clinical Descriptions and Diagnostic Guidelines. Geneva: World Health Organization.

Conflict of Interest Statement: The authors declare that the research was conducted in the absence of any commercial or financial relationships that could be construed as a potential conflict of interest.

Received: 31 March 2011; paper pending published: 01 May 2011; accepted: 23 May 2011; published online: 06 June 2011.

Citation: Dannon PN, Rosenberg O, Schoenfeld N and Kotler M (2011) Acamprosate and baclofen were not effective in the treatment of pathological gambling: preliminary blind rater comparison study. Front. Psychiatry 2:33. doi: 10.3389/fpsyt.2011.00033

This article was submitted to Frontiers in Impulsivity, Compulsivity and Behavioral Dyscontrol, a specialty of Frontiers in Psychiatry.

Copyright (c) 2011 Dannon, Rosenberg, Schoenfeld and Kotler. This is an openaccess article subject to a non-exclusive license between the authors and Frontiers Media SA, which permits use, distribution and reproduction in other forums, provided the original authors and source are credited and other Frontiers conditions are complied with. 